\title{
An urgent call to increase access to evidence-based opioid agonist therapy for prescription opioid use disorders
}

\author{
M. Eugenia Socías MD MSc, Keith Ahamad MD
}

See also page 1240 and www.cmaj.ca/lookup/doi/10.1503/cmaj.160356

Competing interests: None declared.

This article has been peer reviewed.

Correspondence to: Keith Ahamad,kahamad@cfenet. ubc.ca

CMAJ 2016. DOI: 10.1503 /cmaj.160554
A s Fischer and colleagues highlight in a linked Analysis article, ${ }^{1}$ the use of prescription opioids among Canadians has increased dramatically in the last two decades, placing our country behind the United States as the second highest consumer of prescription opioids in the world.

Paralleling this stark increase in prescription opioid use, the prevalence of opioid use disorders and related health and social harms has also increased substantially, with some estimates suggesting that prescription opioid use disorders account for the third highest overall burden of disease attributable to substance use in Canada (after tobacco and alcohol). ${ }^{2}$ To make matters worse, the introduction of fentanyl into the illicit-drug market has drawn attention recently because of a spike in overdose deaths in Canada's largest provinces. Importantly, accidental opioid-related overdose deaths now exceed deaths from alcohol-related motor vehicle collisions in many Canadian provinces, and fentanyl has been involved in many of these deaths. ${ }^{1}$ This alarming rise in overdose deaths underscores the immediate need for a coordinated, evidence-based approach to address the harms associated with untreated opioid use disorders.

Given the complexities of the opioid epidemic, no single solution will prove effective, and concerted efforts will be required to tackle the underlying factors driving this public health crisis. An effective strategy should focus concomitantly on the prevention and early identification of cases of opioid use disorders, as well as ensuring access to

evidence-based comprehensive addiction care that includes pharmacotherapies (i.e., opioid agonist therapy), naloxone for overdose prevention, other harm-reduction approaches (e.g., safe injection sites) and psychosocial supports.

Fischer and colleagues provide an extensive analysis of the current Canadian response to the opioid crisis and suggest specific upstream interventions to address the prescribing side of the problem. ${ }^{1}$ Drawing on lessons from other jurisdictions, the authors make a strong call for tighter restrictions on opioid prescribing through enforceable evidence-based guidelines and mandatory implementation and use of real-time monitoring programs for prescription drugs. We agree that promoting appropriate and safer opioid-prescribing practices should be a key element in the Canadian response to the opioid crisis, particularly to prevent future harms. However, the overdose epidemic related to both prescription and illicit opioids will continue unless we simultaneously target those who have already developed opioid use disorders and expand access to evidence-based addiction treatment.

Unfortunately, major barriers to accessing opioid agonist therapy (e.g., methadone, buprenorphine/ naloxone) continue to exist throughout Canada, which has resulted in a large unmet treatment need. ${ }^{4}$ Limited access to opioid agonist therapy is of concern, because the benefits of these medications are unquestionable (i.e., reductions in illicit drug use, drug-related mortality, and risk of HIV and hepatitis $\mathrm{C}$ virus infection). ${ }^{5,6}$ In particular, buprenorphine/naloxone continues to be underutilized despite ample evidence of its comparable effectiveness and having a better safety profile than methadone. ${ }^{4,5,7}$ Indeed, experiences with this therapy in other countries (e.g., the US and France) have shown that facilitated access to buprenorphine/naloxone through primary care resulted in expanded access to opioid addiction treatment, particularly to many patients who would otherwise not access treatment. ${ }^{7,8}$ Increased uptake of opioid agonist therapy, in turn, has 
been associated with numerous public health benefits, including reductions in drug-related deaths and injecting risk behaviours, and better management of common comorbidities such as HIV infection and mental illness. ${ }^{7,8}$

Factors hampering greater use of buprenorphine/naloxone in Canada include strict regulations for its administration, restricted coverage in many Canadian provinces, limited numbers of trained addiction health care providers and prescribers' concerns about potential diversion. ${ }^{4,7,9}$ For instance, a recent needs assessment in British Columbia documented substantial shortages in the addiction medicine workforce, with substantial geographic variability that ranged from 0 providers per 1000 affected individuals in more rural health authorities to about 20 per 1000 in urban settings. ${ }^{10}$ In recent years, efforts have emerged to address these gaps: scale-up of training programs for addiction medicine and development of evidence-based clinical guidelines for the management of opioid use disorders that recommend buprenorphine/naloxone as a first line pharmacotherapy option. ${ }^{9}$ Although encouraging, these efforts are isolated.

Notably, the lack of a coordinated national response in Canada sharply contrasts with the recent escalation of actions in the US to address the opioid epidemic (e.g., the release of a National Pain Strategy, national guidelines for safer prescription of opioid pain medications, recognition of addiction medicine as an official medical subspecialty by the American Board of Medical Specialties and efforts to expand access to buprenorphine/naloxone). ${ }^{11}$

The US response to the opioid crisis highlights that a comprehensive strategy, which tackles both the roots of the opioid epidemic (e.g., promotion of appropriate and safer opioid prescribing practices through formal training of health care workers and provision of clinical guidelines) and increases and facilitates access to evidence-based addiction care for individuals who have already developed opioid use disorders, ${ }^{3}$ will be needed to curb the opioid epidemic in Canada. In particular, given the wellestablished individual and societal benefits of opioid agonist therapy, 5,7 expanding access to these pharmacotherapies should be a public health priority going forward. Given the benefits of treatment with buprenorphine/naloxone, the remaining restrictions to prescribing and coverage of this drug therapy should be removed and standardized across provinces. Furthermore, building on the success of expanded access to opioid agonist therapy through primary care in other countries ${ }^{7-9}$ and some local successes for HIV infection (e.g., BC's expansion of antiretroviral treatment through provincial cov- erage at no cost to patients that resulted in reductions in HIV-related morbidity, mortality and transmission), ${ }^{12}$ bolder interventions, such as publically funded addiction care that includes free-ofcharge opioid agonist therapy through primary care settings, should also be considered. We need to act now: Canada can no longer afford to be a witness to this devastating epidemic.

\section{References}

1. Fischer B, Rehm J, Tyndall M. Effective Canadian policy to reduce harms from prescription opioids: learning from past failures. CMAJ 2016;188:1240-4.

2. Fischer B, Argento E. Prescription opioid related misuse, harms and interventions in Canada: a review. Pain Physician 2012;15(Suppl 3):ES191-203.

3. Kolodny A, Courtwright DT, Hwang CS, et al. The prescription opioid and heroin crisis: a public health approach to an epidemic of addiction. Annu Rev Public Health 2015;36:559-74

4. Luce J, Strike C. A cross-Canada scan of methadone maintenance treatment policy developments: a report prepared for the Canadian Executive Council on Addictions. Ottawa: Canadian Executive Council on Addictions; 2011.

5. Connery HS. Medication-assisted treatment of opioid use disorder: review of the evidence and future directions. Harv Rev Psychiatry 2015;23:63-75.

6. Mattick RP, Breen C, Kimber J, et al. Buprenorphine maintenance versus placebo or methadone maintenance for opioid dependence. Cochrane Database Syst Rev 2014;(2):CD002207.

7. Duncan LG, Mendoza S, Hansen H. Buprenorphine maintenance for opioid dependence in public sector healthcare: benefits and barriers. J Addict Med Ther Sci 2015;1:31-6.

8. Fatseas M, Auriacombe M. Why buprenorphine is so successful in treating opiate addiction in France. Curr Psychiatry Rep 2007;9:358-64.

9. Moving towards improved access for evidence-based opioid addiction care in British Columbia. Recommendations from the British Columbia Node of the Canadian Research Initiative on Substance Misuse. Vancouver: BC Centre for Excellence in HIV/AIDS, 2016.

10. McEachern J, Ahamad K, Nolan S, et al. A needs assessment of the number of comprehensive addiction care physicians required in a Canadian setting. J Addict Med 2016;10:255-61.

11. Fact sheet: Obama Administration announces additional actions to address the prescription opioid abuse and heroin epidemic [press release]. Washington (DC): The White House: Office of the Press Secretary; 2016 Mar. 29.

12. Montaner JS, Lima VD, Harrigan PR, et al. Expansion of HAART coverage is associated with sustained decreases in HIV/ AIDS morbidity, mortality and HIV transmission: the "HIV Treatment as Prevention" experience in a Canadian setting. PLoS One 2014;9:e87872

Affiliations: British Columbia Centre for Excellence in HIV/ AIDS (Socías, Ahamad), St. Paul's Hospital; Department of Medicine (Socías), University of British Columbia; Department of Family Practice (Ahamad), University of British Columbia, Vancouver, BC

Contributors: Both authors contributed significantly to the manuscript, revised it critically for intellectual content, approved the final version to be published and agreed to act as guarantors of the work.

Acknowledgments: The authors thank Carmen Rock for her administrative assistance. The study was supported by the British Columbia Node of the Canadian Research Initiative on Substance Misuse. M. Eugenia Socías is supported by a Michael Smith Foundation for Health Research Post-Doctoral Fellowship Award and a Canada Addiction Medicine Research Fellowship (US National Institute on Drug Abuse, R25-DA037756). Keith Ahamad is supported by an Embedded Clinician Researcher Salary Award from the Canadian Institutes of Health Research. 